REVIEW ARTICLE

\title{
Methyl-2-formyl benzoate: A Review of Synthesis and Applications
}

Saba Farooq ${ }^{*}$ and Zainab Ngaini

Department of Chemistry, Faculty of Resource Science and Technology, Universiti Malaysia Sarawak, 94300 Kota Samarahan, Sarawak, Malaysia

\begin{abstract}
Methyl-2-formyl benzoate is known as a bioactive precursor in organic synthesis of compounds due to its variety of pharmacological activities namely antifungal, antihypertensive anticancer, antiulcer, antipsychotic and antiviral properties. As an active scaffold, methyl-2-formyl benzoate can be considered as a significant structure and an excellent precursor for the search of new bioactive molecules. Methyl-2-formyl benzoate is synthetically a versatile substrate, which can be used as a raw material for the preparation of medical products. Due to its significant in organic synthesis, the objective of this review is to discuss on the various synthetic routes for the preparation of methyl2-formyl benzoate (via two-step and one-step methodologies) and its importance as precursor for the preparation of compounds with pharmaceutical applications. This review is of importance in synthetic fields and pharmaceutical industry.
\end{abstract}

Keywords: 2-formyl benzoic acid, bioactive molecules, drugs, esterification, precursor, scaffold.

\section{INTRODUCTION}

In a drug discovery process, a readily available and easily prepared precursor has always been an importance choice prior to the synthesis of organic compounds. A good precursor with active functional groups is important at the initial stage of synthesis for functional group manipulation and formation of target molecules. Methyl-2-formyl benzoate (1) is one of the importance compounds which is widely reported as a starting material for the synthesis of various derivatives that capable of interacting with several different receptors for the discovery of new potential drug candidates. Compound $\mathbf{1}$ is mainly consisted of ester and formyl functions at vicinal. Both are important functional groups which are reactive and suitable towards the formation of new compounds.

Methyl-2-formyl benzoate (1) is also known as methyl phthalaldehydate or 2-carboxymethylbenzaldehyde (Fig. 1). It is an essential starting material for the synthesis of many organic compounds via multisteps reactions such as Ugireaction $[1,2]$ and tetrazole photoclick reaction [3]. It is a colorless or yellow colored oily liquid and insoluble in water. Various methods on the preparation of 1 have been reported $[4,5]$. The most commonly reported is via esterification reactions of 2-formylbenzoic acid. Methyl-2-formyl benzoate is a useful starting material that has been used in several important compounds i.e. biisoquinoline tetradentate ligands [6], spiro and fused heterocycles [7], isoquinoline

*Address correspondence to this author at the Department of Chemistry, Faculty of Resource Science and Technology, Universiti Malaysia Sarawak, 94300 Kota Samarahan, Sarawak, Malaysia; Fax: +6082583160;

E-mails: sabafarooq61@yahoo.com/nzainab@unimas.my<smiles>COC(=O)c1ccccc1C=O</smiles>

(1)

Fig. (1). General structure of methyl-2-formylbenzoate prepared via one-step or two-step conditions.

derivatives [8], quinoline based tetracycles [9], phthalides derivatives [10], 3-[alkyl(aryl)thio] isoindolinones [11], ( \pm Crispine A obtained from isoindolinones and tetrahydroisoquinolines[12] and porphyrins [13].

There are various ways reported for the synthesis of $\mathbf{1}$ $[4,14]$ either following one-step or two-step conditions. This brief review is discussing on methods and conditions reported towards the synthesis of $\mathbf{1}$ and some overview of pharmaceutically active compounds prepared using $\mathbf{1}$ as a precusor.

\subsection{Two-Step Methodologies}

A two-step procedure for the synthesis of $\mathbf{1}$ has been widely reported (Scheme 1). Most of the reactions are time consuming and less efficient. It was earlier reported by Rama Rao et al. (1988) via bromination of ester using $\mathrm{Br}_{2} / \mathrm{CCl}_{4}$ followed by the reaction with $\mathrm{AgNO}_{3}$ in THF to afford $80 \%$ yield (method a) [14]. A year later, Murata et al. (1989) performed photoreaction of $o$-carbomethoxyphenyldiazomethane under argon atmosphere to give oxirane intermediate, which further converted into $1(\operatorname{method} b)$ [15]. In 2007, Albert et al. synthesized 1 using slightly longer route 